\title{
Biossurfactantes: potenciais agentes biorremediadores
}

\author{
Biosurfactants: potential bioremediation agents
}

\author{
Sidnei Cerqueira dos Santos ${ }^{1}$ \\ ${ }^{1}$ Universidade Federal do Sul e Sudeste do Pará, Marabá, PA, Brasil
}

\begin{abstract}
Resumo
Os biossurfactantes são moléculas tensoativas que apresentam potencial como agente remediador de ambientes contaminados. A prospecção é uma ferramenta estratégica para identificar inovações tecnológicas. O objetivo deste trabalho foi avaliar as patentes que utilizam biossurfactante no processo de remediação microbiana de ambientes contaminados, para identificar os principais tipos de biossurfactante, os microrganismos produtores e as formas de tratamento. A busca de dados tecnológicos foi realizada nas bases de dados do Questel Orbit, usando-se uma combinação de palavras-chave e códigos da Classificação Internacional de Patentes. Foram selecionadas 76 patentes para análise. A China destaca-se como a maior detentora de patentes. As inovações tecnológicas mais depositadas foram relacionadas com o biossurfactante ramnolipídeo, produzido por Pseudomonas aeruginosa. Esta pesquisa indica que o melhor cenário para desenvolvimento tecnológico é para remediação de metais pesados.
\end{abstract}

Palavras-chave: Microrganismos. Hidrocarboneto. Metais pesados.

\begin{abstract}
Biosurfactants are surfactant molecules that have potential as bioremediation agent for contaminated environments. Prospecting is a strategic tool for identifying technological innovations. The aim of this work was to evaluate patents that use biosurfactant in the microbial remediation process of contaminated environments, to identify the main types of biosurfactant, the producing microorganisms and the forms of treatment. The research for technological data was carried out at the Questel Orbit databases, using the combination of the keywords and codes of the International Patent Classification. We selected 76 patents for analysis. China was the largest patent holder. The most deposited technological innovations were related to the ramnolipid biosurfactant produced by Pseudomonas aeruginosa. This research indicates that the best scenario for technological development is for heavy metal remediation.
\end{abstract}

Keywords: Microorganisms. Hydrocarbon. Heavy metals.

Área Tecnológica: Prospecção Tecnológica, Biotecnologia, Biorremediação. 


\section{Introdução}

Os biossurfactantes são moléculas anfifílicas de superfície ativa, produzidos principalmente por microrganismos (XU et al., 2011). A porção hidrofóbica do biossurfacnate é frequentemente uma cadeia hidrocarbonada, enquanto a porção hidrofílica pode ser iônica (aniônica ou catiônica), não iônica ou anfotérica (DESAI; BANAT, 1997). Estas moléculas possuem a capacidade de reduzir as tensões superficial e interfacial de fluidos imiscíveis, além de apresentarem propriedades de formação de emulsões (BEZZA; CHIRWA, 2017; ELLIOT; SINGHAL; SWIFT, 2010).

Os surfactantes são classificados de acordo com sua composição química e origem microbiana, sendo o tipo, a quantidade e a qualidade dos biossurfactantes influenciados pela natureza do substrato, concentrações de íons no meio de cultura, além das condições de cultivo. As principais classes de biossurfactantes incluem os compostos poliméricos e particulados, ácidos graxos simples, glicolipídeos complexos, lipídeos neutros, lipopeptídeos e lipoproteínas, lipopolissacarídeos e fosfolipídeos (BOGNOLO, 1999; SAIKIA; DEKA, 2015).

Os surfactantes biológicos apresentam propriedades de grande interesse para biorremediação, como baixa toxidade, biodegradabilidade, atividade emulsificante e tensoativa, e a possibilidade de produção a partir de fontes renováveis (PI et al., 2017; SANTOS et al., 2010). A biorremediação é uma técnica que utiliza agentes biológicos, como microrganismos e plantas (fitorremediação) ou seus produtos para reduzir ou eliminar os efeitos adversos causados pelos contaminantes ambientais. O processo metabólico que tem se mostrado mais apto na biorremediação é o microbiano (fungos e bactérias), uma vez que os microrganismos desempenham a tarefa de reciclar a maior parte das moléculas da biosfera, participando dos principais ciclos biogeoquímicos (GAYLARD; BELLINASO; MANFIO, 2005).

Os biossurfactantes podem ser usados para o tratamento de solo, sedimento e água contaminado, tanto com compostos orgânicos e pesticidas, acelerando o processo de degradação e dispersão, quanto com metais pesados, a partir da formação de complexos entre metal e as micelas (DELL'ANNO et al., 2018; GUO et al., 2017; SUN et al., 2019).

A pesquisa de patentes é uma estratégia importante para identificar as inovações e o grau de desenvolvimento dos processos tecnológicos sobre aplicação de biossurfactantes na biorremediação. Os documentos de patente fornecem informações essenciais, como as reivindicações, para identificação da novidade, atividade inventiva e aplicação industrial do produto ou processo a ser protegido (INPI, 2017). Dessa forma, o objetivo deste trabalho foi avaliar as patentes que utilizam biossurfactantes no processo de remediação microbiana de ambientes contaminados, para identificar os principais tipos de biossurfactante, os microrganismos produtores e as formas de tratamento.

\section{Metodologia}

A busca de patentes foi realizada no Questel Orbit, que possui cobertura de dados do Escritório Europeu de Patentes (2019). Esta base de patente possui um sistema integrado e aperfeiçoado de busca, possibilitando tratamento, refino dos dados e exportação com maior grau de liberdade. 
A pesquisa avançada de patentes foi realizada com palavras-chave redigidas na língua inglesa, no título, resumo e nas reivindicações, e dois códigos da Classificação Internacional de Patentes (CIP), correspondentes ao objetivo da busca (Tabela 1). O operador booleano (AND e OR) e o operador de truncagem (*), que permitem encontrar possíveis derivações, foram utilizados na pesquisa.

A busca de patentes foi feita no período de publicação de 1999 a 2018, que corresponde ao desenvolvimento de tecnologias mais recentes, considerando-se os 20 anos de proteção da patente, bem como o período de sigilo de 18 meses (BRASIL, 1996). As patentes selecionadas foram processadas para remover documentos duplicados e que não faziam parte do escopo do trabalho.

Tabela 1 - Estratégia de busca

\begin{tabular}{|c|c|c|c|c|}
\hline ОвJето & Palavras-chave & $\begin{array}{c}\text { RADICAL DAS } \\
\text { PALAVRAS-CHAVE }\end{array}$ & AsSOCIAÇÕES & IPC \\
\hline $\begin{array}{l}\text { Surfactante } \\
\text { Biológico }\end{array}$ & Biosurfactant & biosurfatant* & biosurfactant* AND remed* & $\begin{array}{l}\text { B09C } 1 / 10^{\mathrm{a}} \\
\mathrm{C} 02 \mathrm{~F} 3 / 34^{\mathrm{b}}\end{array}$ \\
\hline \multirow{2}{*}{ Biorremediação } & Remediation & remed* & biosurfactant* AND recover* & $\begin{array}{l}\text { C12N 1/26 } \\
\text { C12R 1/00 }\end{array}$ \\
\hline & Recovery & recover* & $\begin{array}{l}\text { biosurfactant* AND } \\
\text { remed* OR recover* }\end{array}$ & $\begin{array}{l}\text { A62D 3/02e } \\
\text { C02F 3/00f }\end{array}$ \\
\hline
\end{tabular}

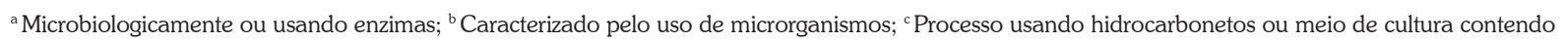
hidrocarbonetos (refinação de óleos de hidrocarbonetos usando microrganismos); ${ }^{\mathrm{d}}$ Microrganismos; ${ }^{e}$ Método biológico, exemplo, processo usando enzimas ou microrganismos; ${ }^{\mathrm{f}}$ Tratamento biológico de água, águas residuais ou esgoto.

Fonte: Elaborado pelo autor deste artigo (2019)

\section{Resultados e Discussão}

A pesquisa realizada na base de dados de patentes do Questel Orbit, nos últimos 20 anos, resultou em 70 patentes, utilizando o radical "biosurfatant" e os códigos B09C1/10 e C02F3/34 da CIP. Essa combinação de palavra-chave e códigos apresentou uma aderência de 43\%, segundo Questel Orbit. Após a mineralização dos dados, foram selecionados 67 documentos.

$\mathrm{Na}$ análise anual foram observados perfis diferentes de depósitos de patentes ao longo dos últimos 20 anos (Figura 1). Este tipo de comportamento é normal no setor de inovação, pois depende de vários fatores, como a demanda por novas tecnologias no mercado, a maturidade do setor, o orçamento para P\&D\&I e a mudança econômica (QUESTEL ORBIT, 2019).

No período de 2011 e 2016 foi observado um crescimento exponencial do número de depósitos de patentes, seguido de declínio (Figura 1). Este crescente desenvolvimento tecnológico de biossurfactantes pode ter relação com a contaminação de cerca de 6,3 milhões de quilômetros quadrados de solo chinês por compostos inorgânicos, no período de oito anos (2005-2013), proveniente de atividade mineradora e da agricultura (JORNAL GGN, 2014). O governo da China reconheceu que $16 \%$ do território chinês está contaminado, incluindo 
metais pesados, como cádmio, mercúrio, arsénico e chumbo (PUBLICO, 2014). Por meio da Figura 1, é possível observar as duas fases deste processo: linear ou exponencial (2011-2016) e declínio (2017 e 2018).

Figura 1 - Evolução anual do número de patentes depositadas

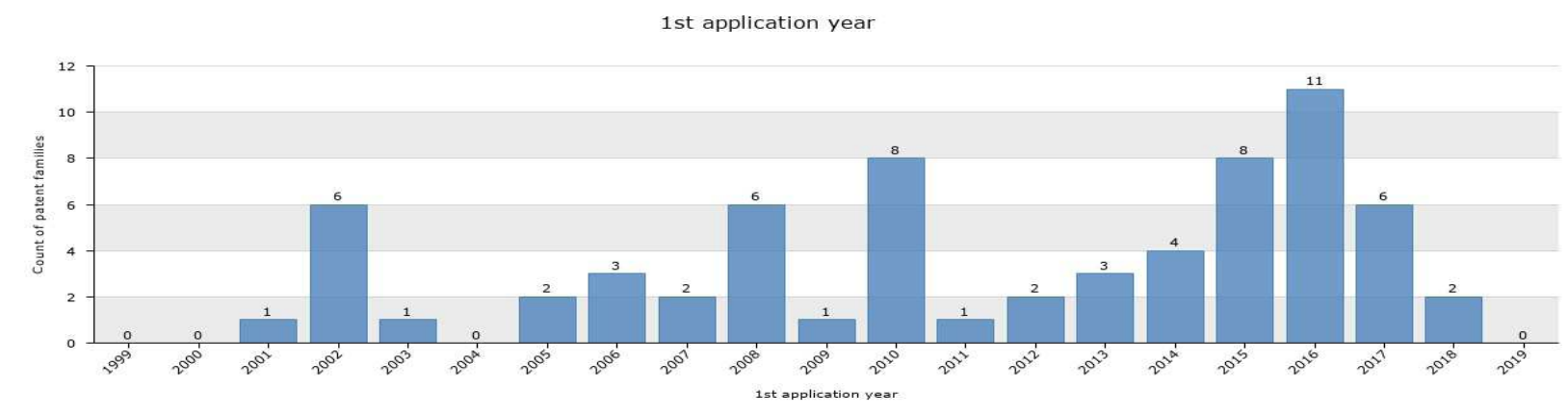

Fonte: Questel Orbit (2019)

A China liderou o ranking dos países depositantes de patentes (Figura 2). Estudo prospectivo recente mostrou que aquele país possui mais de 85 depósitos de patentes relacionadas com a remediação microbiana de metais pesados (SANTOS et al., 2018). Este país da Ásia Oriental possui grande poder econômico, diversidade microbiana (cerca de 30 mil espécies) e capacidade inovadora, que propiciam o desenvolvimento de novas tecnologias para o tratamento ambiental (ZUCOLOTO, 2013), visando a reduzir os níveis de contaminação e garantir o bem-estar da população.

Figura 2 - Depósito de patentes por país, distribuição geográfica e os dez principais países

Patent families by Protection country

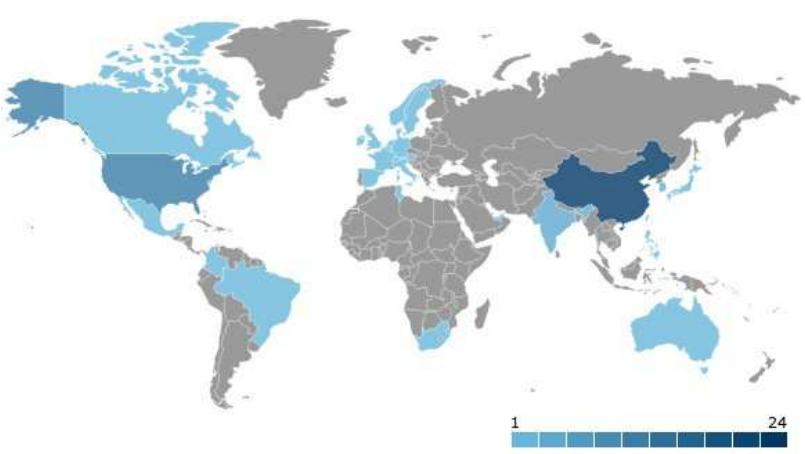

Patent families by Protection country

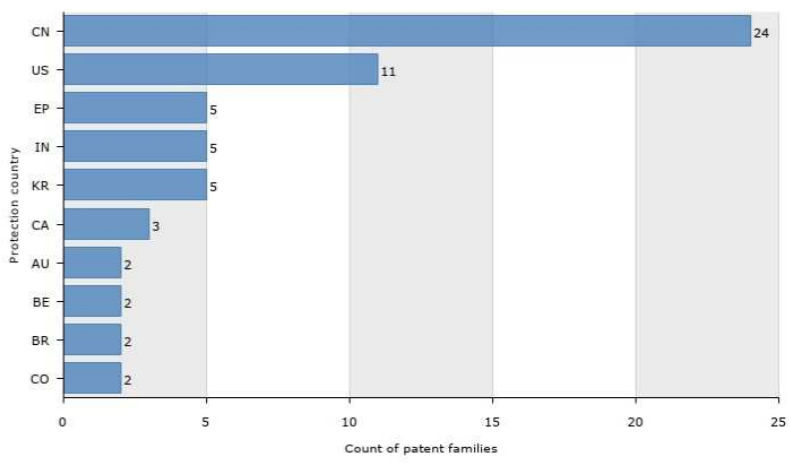

(c) Questel 2019

๑ Questel 2019

Fonte: Questel Orbit (2019)

A Figura 3 apresenta os domínios de tecnologia relacionados com o tema da pesquisa, baseados em agrupamentos de código do IPC (QUESTEL ORBIT, 2019). O resultado mostrou que existe grande diversidade e especificidade em três domínios: Tecnologia Ambiental, Biotecnologia e Química de Materiais Básicos, permitindo identificar as possíveis oportunidades de negócio (core business), bem como outras potenciais aplicações dos biossurfactantes. 
Figura 3 - Tecnologias dominantes

Patent families by Technology domain

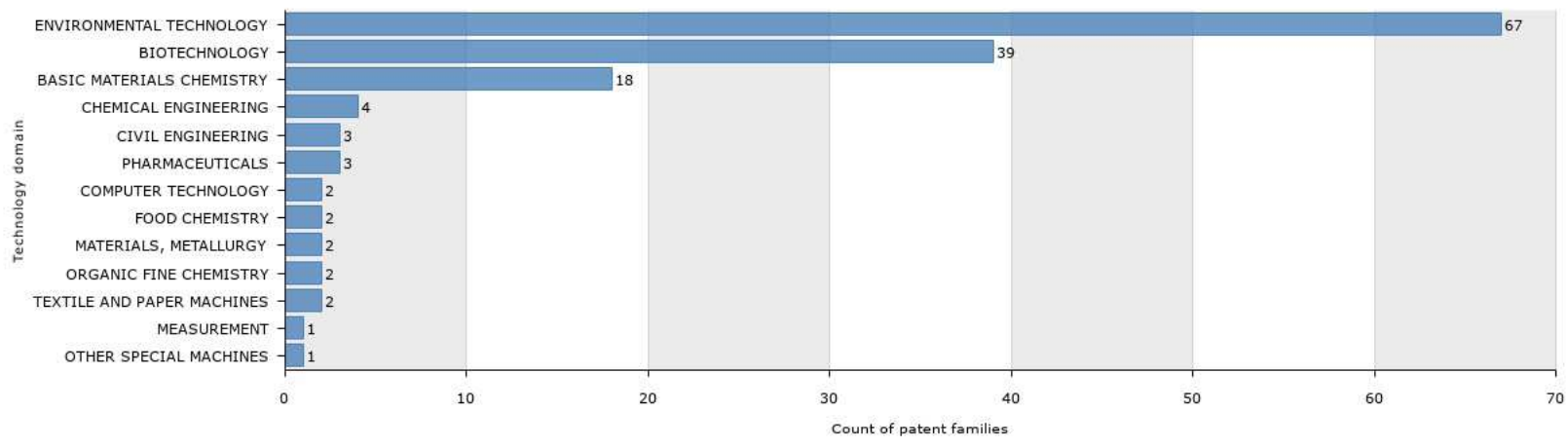

๑Questel 2019

Fonte: Questel Orbit (2019)

As patentes foram depositadas por pessoas jurídicas, principalmente empresas, institutos de pesquisa e universidades (Figura 4). Mesmo a China sendo a maior detentora das patentes, a empresa americana DuPont (E. I. du Pont de Nemours and Company) foi a que apresentou o maior número de depósitos de patente (Figura 4). Esta foi formada em 2017, a partir de uma fusão entre a Dow Chemical Company e a DuPont, e é considerada a segunda maior empresa química do mundo (CHEPKEMOI, 2019). Esta empresa tem trabalhado com inovação há mais de 200 anos e apresenta no seu portfólio o slogan "Ciência e engenharia são a base de nossas inovações essenciais". A DuPont tem desenvolvido uma combinação de recursos em agricultura, biotecnologia, química e ciência de materiais para solução de problemas ambientais (DUPONT, 2019).

Figura 4 - Patentes por depositante

Patent families by Assignees

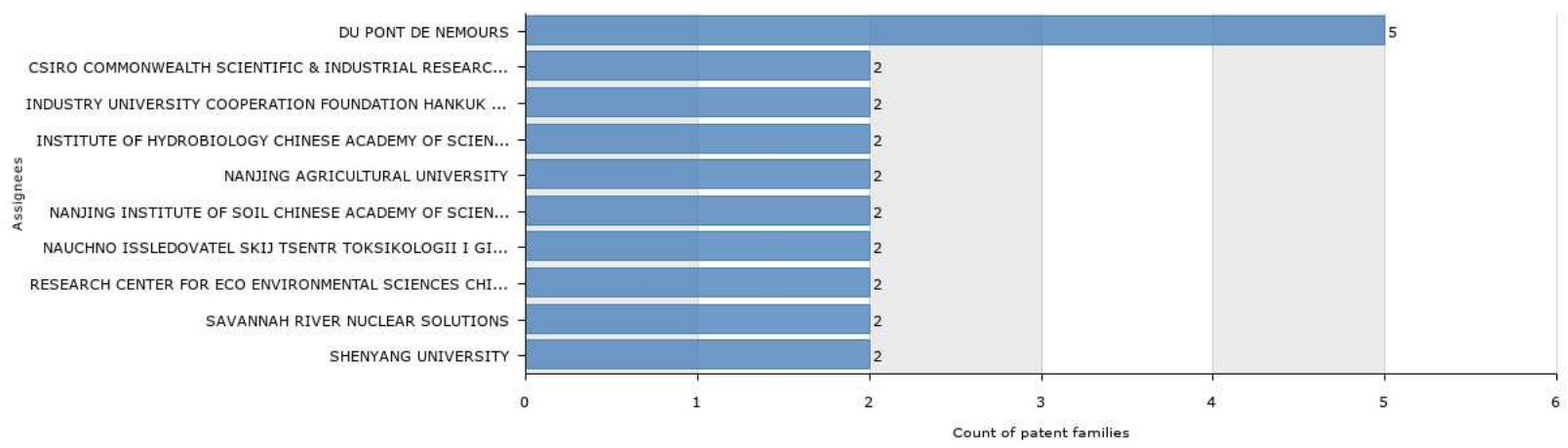

๑ Q Questel 2019

Fonte: Questel Orbit (2019)

A Tabela 2 apresenta classes, tipos, microrganismos produtores de biossurfactantes e suas principais aplicações na biorremediação de ambientes contaminados. A principal classe de biossurfactante foi o glicolipídeo. O glicolipídeo é um dos surfactantes microbianos mais estudados, sendo que o composto mais conhecido é o ramnolipídeo (CHRZANOWSKI; ŁAWNICZAK; CZACZYK, 2012). O ramnolipídeo possui a capacidade de reduzir a tensão superficial da água 
para 25 a $30 \mathrm{mN} / \mathrm{m}$ e a tensão interfacial do n-hexadecano para $1 \mathrm{mN} / \mathrm{m}$ (QAZI et al., 2013), bem como formar emulsões. Estas propriedades, aliadas à baixa toxicidade e biodegradabilidade, tornam essa molécula um eficiente agente biorremediador de áreas impactadas por compostos orgânicos e metais pesados.

Tabela 2 - Classificação, microrganismos produtores e aplicação dos biossurfactantes usados na biorremediação

\begin{tabular}{|c|c|c|c|c|}
\hline Classe & TIPO & $\begin{array}{l}\text { MicRORGANISMOS } \\
\text { PRODUTORES }\end{array}$ & APLICAÇÃo & REFERÊNCIAS \\
\hline \multirow[t]{2}{*}{ Glicolipídeo } & Ramnolipídeo & $\begin{array}{l}\text { Pseudomonas } \\
\text { aeruginosa, } \\
\text { Pseudomonas sp. }\end{array}$ & $\begin{array}{l}\text { Acelerar a degradação e dispersão } \\
\text { de compostos orgânicos, como } \\
\text { hidrocarbonetos e derivados; } \\
\text { emulsificação de hidrocarbonetos } \\
\text { e óleos vegetais; recuperação } \\
\text { avançada de petróleo; remoção } \\
\text { de metais pesados. }\end{array}$ & $\begin{array}{l}\text { CN102771221; } \\
\text { CN105598159; } \\
\text { CN106001097; } \\
\text { WO201655591; } \\
\text { CN101948793B; } \\
\text { CN101780465B; } \\
\text { CN107497364; } \\
\text { CN101948786B; }\end{array}$ \\
\hline & Soforolipídeo & Candida tropicalis & $\begin{array}{c}\text { Recuperação de petróleo } \\
\text { e derivados de ambientes } \\
\text { contaminados; tratamento de } \\
\text { esgoto; recuperação de solo } \\
\text { contaminação por metais pesados. }\end{array}$ & CN105567580 \\
\hline $\begin{array}{l}\text { Lipopeptídeo e } \\
\text { Lipoproteína }\end{array}$ & $\begin{array}{c}\text { Fengicina } \\
\text { Iturina }\end{array}$ & $\begin{array}{l}\text { Bacillus subtilis, } \\
\text { Bacillus } \\
\text { amyloliquefaciens, } \\
\text { Bacillus sp. } \\
\\
\text { Bacillus } \\
\text { amyloliquefaciens, } \\
\text { Bacillus sp. }\end{array}$ & $\begin{array}{l}\text { Acelerar a degradação de } \\
\text { hidrocarbonetos e pesticidas; } \\
\text { remoção de metais pesados de solo } \\
\text { contaminado, sedimentos e água. }\end{array}$ & $\begin{array}{l}\text { WO201744953; } \\
\text { TN2015000213; } \\
\text { CN102652957; } \\
\text { WO201697857; } \\
\text { EP1436234; } \\
\text { WO201611805 }\end{array}$ \\
\hline
\end{tabular}

*Não informado.

Fonte: Elaborado pelo autor deste artigo (2019)

A partir da mineralização dos dados, foi possível observar que as principais formas de utilização dos biossurfactantes estão associadas a consórcios microbianos, sendo que 90,8\% das patentes estão relacionadas com o processo de biodegradação de pesticidas e compostos orgânicos, como petróleo e seus derivados, e com a recuperação avançada de petróleo. O processo de remediação de compostos orgânicos com biossurfactante aumenta a solubilidade e a biodisponibilidade do contaminante, que podem ser degradados pelos microrganismos (POURFADAKARI et al., 2019; SUN et al., 2019). Os outros 9,2\% dos documentos foram referentes ao tratamento de ambientes contaminados por metais pesados. Nesse processo, os biossurfactantes formam complexos com os metais pesados. As ligações entre metal e as micelas dos biossurfactantes podem ocorrer por atração entre tensoativos aniônicos e formas metálicas não iónicas, ou por formação de quelatos na superfície das micelas (BANAT et al., 2010; RUFINO et al., 2011). Dessa forma, os metais ficam retidos nas micelas por meio de interações eletrostáticas, podendo ser recuperados por meio de técnicas de separação por membranas (KITAMOTO et al., 2001).

O resultado mostrou que o ramnolipídeo, produzido por Pseudomonas aeruginosa, foi o principal surfactante utilizado nos processos de biorremediação (Figura 5). Relatos da literatura mostram que a espécie $P$. aeruginosa produtora de biossurfactante (ramnolipídeo) é a mais estudada e que tem apresentado resultados promissores para o desenvolvimento de tecnologias 
de remediação ambiental (NITSCHKE; COSTA; CONTIERO, 2011; PATOWARY et al., 2018; POURFADAKARI et al., 2019).

Urum e Pekdemir (2004) relataram que o tratamento com ramnolipídeo removeu aproximadamente $80 \%$ do petróleo bruto do solo $(87,5 \mathrm{mg} / \mathrm{kg})$. Bordas, Lafrance e Villemur (2005) observaram que 5,0 g/L de ramnolipídeo foram capazes de remover $70 \%$ de hidrocarbonetos aromáticos policíclicos (HAPs) do solo. Li et al. (2019) também mostraram que a linhagem Pseudomonas sp. LKY-5 produtora de ramnolipídeo degradou entre 28 a $42 \%$ de HAPs. Patowary et al. (2018) mostraram que Pseudomanas aeruginosa produtora de ramnolipídeo degradou entre 60 a $80 \%$ de HAPs.

Por outro lado, Mulligan et al. (2001) mostraram que 0,5\% de ramnolipídeo produzidos por $P$. aeruginosa foram capazes de remover $65 \%$ de $\mathrm{Cu}$ e $18 \%$ de $\mathrm{Zn}$ em uma única lavagem, a partir do sedimento contaminado com $110 \mathrm{mg} / \mathrm{kg}$ e $3.300 \mathrm{mg} / \mathrm{kg}$, respectivamente. Juwarkar et al. (2008) observaram que a concentração de 0,1\% de di-ramnolipídeo produzido pela linhagem $P$. aeruginosa $\mathrm{BS} 2$ removeu $88 \%$ de $\mathrm{Cu}$ e $80 \%$ de $\mathrm{Pb}$ do solo contaminado com 500 ppm e 1000 ppm, respectivamente. Wang e Mulligan (2004) relataram que o ramnolipídeo foi capaz de remover 61,7\% de Cd e 51\% de Ni. Aşçi, Nurbaş e Açikel (2010) recuperaram cerca de $91 \%$ de $\mathrm{Cd}$ e $87 \%$ de $\mathrm{Zn}$ usando $25 \mathrm{mM}$ de ramnolipídeo.

Figura 5 - Principais tipos de biossurfactantes usados no processo de biorremediação e de microrganismos produtores

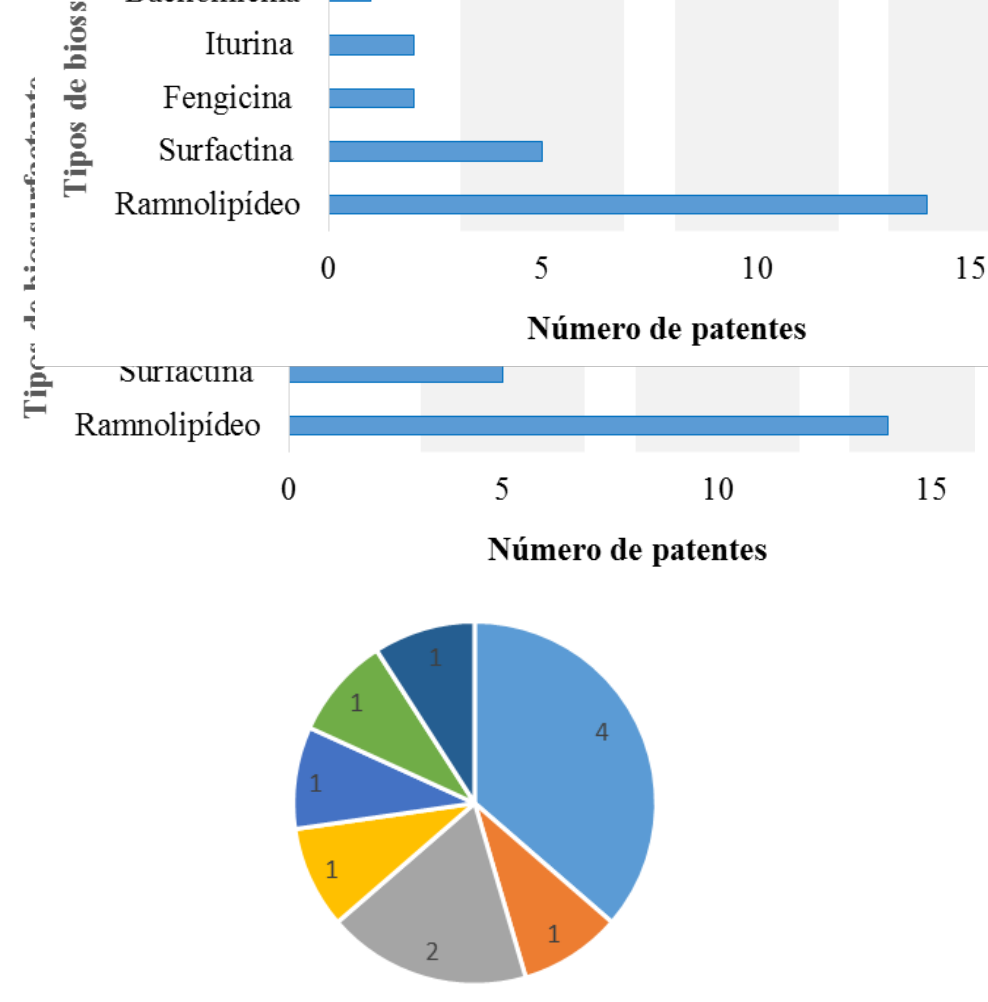

$$
\begin{array}{ll}
\text { " Pseudomonas aeruginosa } & \approx \text { Pseudomonas sp. } \\
\text { " Bacillus subtilis } & \approx \text { Bacillus amyloliquefaciens } \\
\text { - Bacillus pumilus } & \quad \text { Bacillus sp. } \\
\text { - Candida tropicalis } &
\end{array}
$$

Fonte: Elaborada pelo autor deste artigo (2019) 


\section{Considerações Finais}

Os resultados apontam que as principais invenções tecnológicas utilizaram o ramnolipídeo, produzido por Pseudomonas aeruginosa, como agente biorremediador de compostos orgânicos e de metais pesados. Esta pesquisa também indica que há um cenário estratégico para o desenvolvimento de processos de remediação microbiana para recuperação de áreas impactadas por metais pesados, tendo em vista o baixo número de depósitos de patente, os riscos cada vez mais frequentes de incidentes ambientais em todo o mundo, provenientes da atividade mineradora $e$ de contaminação do solo e água com agrotóxicos, principalmente no Brasil, após a aprovação do novo marco regulatório para agrotóxicos.

\section{Referências}

AŞÇI, Y.; NURBAŞ, M.; AÇIKEL, Y.S. Investigation of sorption/desorption equilibria of heavy metal ions on/from quartz using rhamnolipid biosurfactant. J. Environ. Manage, v. 91, p. 724-731, 2010.

BEZZA, F. A.; CHIRWA, E. M. N. The role of lipopeptide biosurfactant on microbial remediation of aged polycyclic aromatic hydrocarbons (PAHs)-contaminated soil. Chem. Eng. J., v. 309, p. 563576, 2017.

BANAT, I. M.; FRANZETTI A.; GANDOLFI I, Bestetti, G.; Martinotti, M. G.; Fracchia, L.; Smyth, T. J.; Marchant, R. Microbial biosurfactants production, applications and future potential. Appl. Microbiol. Biotechnol., v. 87, n. 2, p. 427-444, 2010.

BOGNOLO, G. Biosurfactants as emulsifying for hydrocarbons. Physicochem. Eng. Aspects, v. 152, p. 41-52, 1999.

BORDAS, F; LAFRANCE, P.; VILLEMUR, R. Conditions for effective removal of pyrene from an artificially contaminated soil using Pseudomonas aeruginosa 57SJ rhamnolipids. Environ. Poll., v. 138, p. 69-76, 2005.

BRASIL. Lei n. 9.279, de 14 de maio de 1996. Regula direitos e obrigações relativos à propriedade industrial. Diário Oficial [da] República Federativa do Brasil. Brasília, DF, 14 maio 1996. Disponível em: http://www.planalto.gov.br/ccivil_03/Leis/L9279.htm. Acesso em: 31 jul. 2019.

CHEPKEMOI, J. “The world's largest chemical companies”. WorldAtlas, 2019. Disponível em: worldatlas.com/articles/which-are-the-world-s-largest-chemical-producing-companies.html. Acesso em: 31 jul. 2019.

CHRZANOWSKI, L.; ŁAWNICZAK, L.; CZACZYK, K. Why do microorganisms produce rhamnolipids? World J. Microbiol. Biotechnol., v. 28, n. 401-419, 2012.

DELLANNO, F.; SANSONE, C.; IANORA, A.; DELLANNO, A. Biosurfactant-induced remediation of contaminated marine sediments: Current knowledge and future perspectives. Marine Environmental Research, 2018. Doi: 10.1016/j.marenvres.2018.03.010.

DESAI, J. D.; BANAT, I. M. Microbial production of surfactants and their commercial potential. Micriobiol. Mol. Rev., v. 61, p. 47-64, 1997.

DUPONT. Disponível em: www.dupont.com. Acesso em: 10 jul. 2019. 
ELLIOT, R.; SINGHAL, N.; SWIFT, S. Surfactants and bacterial bioremediation of polycyclic aromatic hydrocarbon contaminated soil - unlocking the targets. Crit. Rev. Environ. Sci. Technol., v. 41, p. 78-124, 2010.

EUROPEAN PATENT OFFICE (EPO). 2019. Disponível em: https://www.epo.org/index.html. Acesso em: 31 jul. 2019.

GUO, YI-MING; LIU, YUN-GUO; LI, H.; ZHENG, AI-BING; TAN, XIAO-FEI; ZHANG, MING-MING. Remediation of $\mathrm{Pb}$-contaminated port sediment by biosurfactant from Bacillus sp. G1 Yi-ming.

Trans. Nonferrous Met. Soc. China, v. 27, p. 1.385-1.393, 2017.

GAYLARDE, C. C.; BELLINASO, M. L.; MANFIO, G. P. Biorremediação: aspectos biológicos e técnicos da biorremediação de xenobióticos. Biotecnologia Ciência e Desenvolvimento, v. 34, p. 36-43, 2005.

INSTITUTO NACIONAL DA PROPRIEDADE INDUSTRIAL (INPI). Portal virtual. 2017. Disponível em: http://www.inpi.gov.br/. Acesso em: 01 ago. 2019.

JORNAL GGN. [Base de dados - Internet]. China anuncia que enorme área de seu território está com solo contaminado; 2014. Disponível em: http://jornalggn.com.br/noticia/china-anunciaque-enorme-area-de-seu-territorio-esta-com-solo-contaminado. Acesso em: 18 jun. 2019.

JUWARKAR, A. A.; DUBEY, K. V.; NAIR, A.; SINGH, S. K. Bioremediation of multi-metal contaminated soil using biosurfactant - a novel approach. Indian J. Microbiol., v. 48, p. 142-146, 2008.

KITAMOTO, D.; YANGISHITA, H.; NAKAIWA, M.; NAKANE, T.; AKIYA, T. Remarkable antiagglomeration effect of a yeast biosurfactant, diacylmannosylerythritol, on ice-water slurry for cold thermal storge. Biotechonology, v. 17, p. 362-365, 2001.

LIA, L.; SHENC, X.; ZHAOD, C.; LIUD, Q.; LIUB, X.; WUB, Y. Biodegradation of dibenzothiophene by efficient Pseudomonas sp. LKY-5 with the production of a biosurfactant. Ecotoxicology and Environmental Safety, v. 176, p. 50-57, 2019.

MULLIGAN, C. N.; YONG, R. N.; GIBBS, B. F. Heavy metal removal from sediments by biosurfactants. J. Hazard. Mater, v. 85, p. 111-125, 2001.

NITSCHKE, M.; COSTA, S. G. V. A. O.; CONTIERO, J. Rhamnolipids and PHAs: recent reports on Pseudomonas-derived molecules of increasing industrial interest. Process Biochem., v. 46, p. 621-630, 2011.

PATOWARYA, R.; PATOWARYA, K.; KALITAB, M. C.; DEKA S. Application of biosurfactant for enhancement of bioremediation process of crude oil contaminated soil. International Biodeterioration \& Biodegradation, 2018. Disponível em: https://doi.org/10.1016/j. ibiod.2018.01.004. Acesso em: 25 jul. 2019.

PI, Y.; CHEN, B.; BAO, M.; FAN, F.; CAI, Q.; ZE, L.; ZHANG, B. Microbial degradation of four crude oil by biosurfactant producing strain Rhodococcus sp. Bioresource Technol., v. 232, p. 263-269, 2017.

POURFADAKARI, S.; AHMADI, M.; JAAFARZADEH, N.; TAKDASTAN, A.; NEISI A. A.; GHAFARI, S.; JORFI, S. Remediation of PAHs contaminated soil using a sequence of soil washing with biosurfactant produced by Pseudomonas aeruginosa strain PF2 and electrokinetic oxidation of desorbed solution, effect of electrode modification with $\mathrm{Fe}_{3} \mathrm{O}_{4}$ nanoparticles. Journal of Hazardous Materials, v. 379, p. 1-14, 2019.

PUBLICO. Mais de 16,1\% do solo chinês está contaminado, reconheceu o Governo. 2014. Disponível em: https:/www.publico.pt/2014/04/18/mundo/noticia/mais-de-161-do-solo-chinesestacontaminado-reconheceu-o-governo-1632709. Acesso em: 03 jul. 2019. 
QAZI, M. A.; QURESHI, G. D. HAMEED, A.; AHMED, D. Yeast extract as the most preferable substrate for optimized biosurfactant production by rhlB gene positive Pseudomonas putida SOL-10 Isolate. J. Bioremed. Biode., v. 4, n. 7, p. 1-10, 2013.

QUESTEL ORBIT. 2019. Disponível em: https://www38.orbit.com/. Acesso em: 10 jul. 2019.

RUFINO, R. D.; RODRIGUES, G. I. B.; CAMPOS-TAKAKI, G. M.; SARUBBO, L. A.; FERREIRA, S. R. M. Application of a yeast biosurfactant in the removal of heavy metals and hydrophobic contaminant in a soil used as slurry barrier. Applied and Environmental Soil Science, v. 2011, p. 1-7, 2011. Doi:10.1155/2011/939648.

SAIKIA, R. R.; DEKA, S. Biosurfactants: structure, function and their properties. Int. J. Educ., Sci. Res. Rev., v. 2, p. 5-20, 2015.

SANTOS, S. C.; FERNANDEZ, L. G.; ROSSI-ALVA, J. C.; ROQUE, M. R. A. Evaluation of substrates from renewable-resources in biosurfactants production by Pseudomonas strains. Afr. J. Biotechnol., v. 9, p. 5.704-5.711, 2010.

SANTOS, S. C.; CASTRO, D. C. M.; ASSUNÇÃO, P. S.; SANTOS, T. L.; QUINTELLA, C. M. Mapeamento tecnológico de processos microbianos aplicados na biorremediação de metais pesados. Cadernos de Prospecção, v. 11, n. 5, p. 1.740-1.751, 2018.

SUN, S.; WANG, Y.; ZANG, T.; WEI, J.; WU, H.; WEI, C.; QIU, G.; LI, F. A biosurfactant producing Pseudomonas aeruginos S5 isolated from coking wastewater and its application for bioremediation of polycyclic aromatic hydrocarbons. Bioresource Technology, 2019. Doi: https://doi.org/10.1016/j. biortech.

URUM, K.; PEKDEMIR, T. Evaluation of biosurfactants for crude oil contaminated soil washing. Chemosphere, v. 57, p. 1.139-1.150, 2004.

WANG, S.; MULLIGAN, C. N. Rhamnolipid foam enhanced remediation of cadmium and nickel contaminated soil. Water Air Soil Pollut., v. 157, p. 315-330, 2004.

XU, Q.; NAKAJIMA, M.; LIU, Z.; SHIINA, T. Biosurfactants for microbubble preparation and application. Int. J. Mol. Sci., v. 12, p. 462-475, 2011.

ZUCOLOTO, F. G. Propriedade Intelectual e aspectos regulatórios em biotecnologia: China. In: ZUCOLOTO, F. G.; FREITAS, R. E. (org.). Propriedade Intelectual e aspectos regulatórios em biotecnologia. Rio de Janeiro: Instituto de Pesquisa Econômica Aplicada, 2013. Disponível em: http://protec.org.br/uploads/paginas/file/Propriedade\%20Intelectual\%20e\%20aspectos\%20 regulat\%C3\%B3rios\%20em\%20biotecnologia.pdf. Acessado em: 17 set. 2017.

\section{Sobre o Autor}

\section{Sidnei Cerqueira dos Santos}

E-mail: sidnei.cerqueiradossantos@gmail.com

Doutor em Biotecnologia pela UECE (2013).

Endereço profissional: Universidade Federal do Sul e Sudeste do Pará, Instituto de Estudos em Saúde e Biológicas, Avenida dos Ipês, s/n, Cidade Universitária, Loteamento Cidade Jardim, Marabá, PA. CEP: 68500-000. 